Darko Tanaskovic

Beograd

\title{
MAȘDAR ARABO ALLA LUCE DELLA TEORIA DELLE PARTI DEL DISCORSO
}

La divisione delle unità linguistiche in un numero determinato di categorie o di classi è una delle operazioni linguistiche storicamente più antiche. Uno studio linguistico sistematico non si sarebbe potuto nemmeno pensare senza un raggruppamento grammaticale delle unità lessicali della lingua. Questa suddivisione dovrebbe esprimere i rapporti formali, funzionali e semantici esistenti all'interno del sistema linguistico, soddisfacendo contemporaneamente determinate categorie filosofiche e logiche generalmente accettate. In tal modo la divisione ideale delle parole in categorie dovrebbe esprimere il legame tra la lingua naturale, il mondo delle rappresentazioni umane e della realtà obiettivamente esistente nella quale agisce l'umanità. Tenuto conto della complessità dei compiti posti dinanzi alla teoria delle parti del discorso non ci meraviglia la constatazione che al suo perfezionamento si sia lavorato intensamente e tenacemente; d'altra parte è altrettanto chiaro che anche la più perfetta delle suddivisioni delle parole in categorie finora compiuta non può soddisfare sotto tutti gli aspetti, nell'ambito di una sola lingua e tanto più, quindi, su un piano di linguistica generale. Tuttavia gli sforzi che sono stati compiuti allo scopo di raggruppare le unità linguistiche nel modo più adeguato e più comprensivo possibile, tenendo conto di tutti i criteri utili per il compimento di questa classificazione hanno considerevolmente contribuito allo sviluppo della linguistica. Infatti lavorando alla classificazione delle parole è stato possibile acquisire una più chiara visione d'assieme delle loro numerose caratteristiche che possono sfuggire all'occhio del linguista quando questi si limiti ad osservare isolatamente le unità linguistiche. E proprio questo momento è d'importanza essenziale per la conoscenza della struttura di una lingua, perché, come è stato osservato da de Saussure, «la parola, nonostante tutte le difficoltà che essa crea quando dobbiamo definirla, è un'unità che $s^{\prime}$ impone allo spirito, qualcosa di centrale nel meccanismo della lingua.»1

La divisione tradizionale delle parole in categorie, che, nelle sue diverse varianti, possiamo considerare dominante nella linguistica mon-

${ }^{1}$ v. F. de Saussure, Cours de linguistique générale, Paris, 1965, 154. 
diale, è ben lungi dal dare risposte soddiafacienti ad un livello di linguistica generale, in quanto è costruita sulla base di materiale offerto principalmente dalle lingue indoeuropee fra le quali prevalgono quelle che appartengono alle cerchia della civiltà europea. I fondamenti di questa divisione sono stati posti dai filosofi e dai linguisti dell'antichità e precisamente da Platone e da Aristotele, il quale compì la prima divisione articolata delle parole in tre classi, nome, verbo e particella. Questa divisione ha conservato un'importanza chiave nel corso dell'intera storia della linguistica, sebbene le generazioni successive di studiosi, in armonia con le proprie scelte teoretiche e dipendentemente dalla lingua di cui si sono occupati, abbiano elaborato tutta una serie di classificazioni fra di loro più o meno differenziate. Nel corso di questa lunga storia della classificazione delle unità linguistiche si è andato cristallizando un numero notevole di criteri classificatori che potrebbero essere suddivisi in tre gruppi pricipali. Abbiamo così criteri morfologici, sintattici e semantici, inoltre dal collegamento di quello sintattico con quello semantico si è formato un criterio funzionale con caratteristiche di comprensività particolarmente accentuate. Sebbene con lo sviluppo della linguistica sono state proposte impostazioni diverse di questa delicata questione, tuttavia, fatte le debite limitazioni, è possibile affermare che abbiano prevalso i criteri formali di classificazione, che talvolta sono stati portati agli estremi di formalità, con la conseguenza che i sistemi costruiti, per quanto all'apparenza siano ordinati e logici, in effetti risultano essere in disarmonia con la struttura reale della lingua e col funzionamento di questa. ${ }^{2}$ In tutte le lingue esistono molte unità che non possono essere inserite nelle «parti del discorso» date, perché non soddisfano alcuni dei criteri previsti per le singole categorie, oppure, più esattamente, sono poche le parole la cui struttura risulta essere, per così dire, in tutto e per tutto, «su misura» rispetto alle classificazioni assunte. Sono molti i linguisti che hanno espresso la loro sfiducia sulle possibilità di ampliamento del significato e sull'estensibilità dell'ambito teorico presentate da ogni classificazione delle unità linguistiche costruita su basi tradizionali. Persino un grande estimatore della regolarità e della normatività nella ricerca linguistica, qual'è il neogrammatico Hermann Paul, ha scritto - nei suoi Principi della storia della lingua - «La classificazione delle parti del discorso, che nelle sue linee generali risale ancora ai grammatici antichi, non si basa su principi logici coerentemente applicati ${ }^{3}$ mentre un linguista di orientamento metodologico completamente diverso, Leonard Bloomfield, concluse che «per una lingua qual'è quella inglese è impossibile concepire un sistema soddisfacente di divisione delle parole in

2 Particolarmente adatto come esempio è la classificazione strettamente formale data dal linguista russo F. F. Fortunatov e dai suoi seguaci, specialmente M. N. Peterson, v. M. N. Peterson, Sooremennyj russkij jazyk, Moskva, 1929.

3 v. H. P., Prinzipien der Sprachgeschichte, Halle, 1880, 200. 


\section{Darko Tanaskobić}

categorie, perché l'elenco delle nostre categorie dipenderà dalle funzioni che considereremo più importanti». Abbiamo l'impressione che il problema della classificazione delle unità linguistiche si collochi nel punto d'incontro di due desideri, o meglio, di due esigenze delle quali i linguisti sono, generalmente, consapevoli: da una parte una descrizione il più possibile precisa dello strato superficiale dell'espressione linguistica, dall'altra l'accertamento di alcune leggi generali e categoriali che colleghino la lingua con il mondo e con la rappresentazione che di esso ha ciascun individuo, vale a dire col pensiero umano, ovvero la conoscenza «della strutturta profonda» della lingua, per servirci, con una certa libertà, dalla terminologia usata dalla grammatica generativa. ${ }^{5} \mathrm{Un}$ accostamento integrale alla classificazione delle unità linguistiche che soddisfi entrambe le tendenze ricordate finora non ha potuto ricevere una sua formulazione completa ed è incerto se potrà mai trovarla. È però sicuro che le conquiste della linguistica contemporanea creano le condizioni per andare oltre le semplici proposte di modificazioni insignificanti delle divisioni tradizionali, per poter dedicare un'attenzione maggiore alla distinzione delle categorie generali nelle quali si inseriscono i significati lessicali, distinti dalle accidenze, ${ }^{6}$ di una determinata categoria di parole di una lingua. E proprio questo è l'orientamento di ricerca al quale si è applicato in modo particolare il famoso linguista e lessicologo russo Leonid Ščerba. ${ }^{7}$ Naturalmente la divisione tradizionale non è da rigettare acriticamente ma è piuttosto necessario permearla di un suo più profondo significato ed allargarla sul piano della linguistica generale, il che sottintende l'inclusione di conoscenze derivate dall'analisi delle lingue non indoeuropee da aggiungere agli evidenti meriti acquisiti dell'indoeuropeistica. «La soluzione del problema suscitato dalla teoria delle parti del discorso non consiste nell'accertamento delle loro caratteristiche morfologiche esteriori, che mutano da lingua a lingua, ma piuttosto nella scoperta di qualcosa di più profondo e di più stabile situato sul piano del contenuto e nel campo della funzione e che sia collegato a normatività generali (per quanto apparentemente differenti) della lingua umana

4 v. L. B., Language, New York, 1966, 269.

5 v. linteressante capitolo intitolato «The parts of speech» nel libro J. Lyons, Introduction to Theoretical Linguistics, Cambridge, 1971, 317-333. Il linguista danese V. Brøndal, alla ricerca di una teoria universale delle parti del discorso, abbandona completamente il procedimento induttivo per appoggiare il suo discorso sulle categorie logiche, v. O. Ducrot et Tz. Todorov, Dictionnaire encyclopédique des sciences du langage, Paris, 1972, 263-.265.

6 Il termine è tratto dal libro Iu. S. Maslov, Voedenie o jazykoznanie, Moskva, $1975,203$.

7 v. L. V. Ščerba, $O$ častjah reči 0 russkom jazyke, in Jazykooaja sistema $i$ rečevaja dejatel'nost, Moskva, 1974, 77-100; O. S. Ahmanova al primo posto fra i criteri della divisione delle parole in categorie pone: «un significato comune (astratto, categoriale) che accompagna il concreto significato lessicale di una parola data», v. Slooar' lingoističeskih terminoo, Moskva, 1966, 511. 
e del pensiero umano stesso» conclude correttamente il linguista russo Iu. S. Maslov. ${ }^{8}$

I limiti della divisione tradizionale delle parole in parti del discorso si rivelano in tutta la loro evidenza sull'esempio del mașdar arabo, forma specifica che presenta allo stesso tempo proprietà verbali e nominali, tanto che non è facile inserirlo in nessuna delle classi di parole esisenti. Il mașdar viene per lo più definito come «infinito arabo» oppure come «nome verbale». E già questi dati alludono alla sua natura difficilmente afferrabile. Da un punto di vista formale il mașdar apparterrebbe alla classe dei nomi, perché ha la forma di nome, viene declinato secondo i casi, riceve l'articolo determinativo, può comparire al plurale e così via. Anche la sintassi ci orienta verso la natura nominale del mașdar, perché esso può comparire quasi in tutte le combinazioni caratteristiche del nome (come oggetto diretto, con preposizioni, ecc.). Tuttavia non è possibile inserire semplicemente il mașdar fra i nomi, perché la sua semantica verbale ha proprietà che non possono essere trascurate. Il mașdar, in effetti. appartiene a quelle categorie di parole che taluni chiamano «verboidi» in quanto uniscono le proprietà del verbo con quelle di altre categorie di parole, prima di tutto dei nomi. In senso lato si può affermare che il mașdar esprime l'azione verbale in modo astratto, cioè senza ricollegarla ad un agente determinato, ad un oggetto, ad un tempo o a un modo d'esecuzione fissati. Nella grammatica araba classica il mașdar a una collocazione abbastanza chiaramente definita nell'ambito della divisione tripartita delle parole in categorie. Questa classificazione è identica a quella di Aristotele ed è servita come uno degli argomenti fondamentali per l'asserzione che la grammatica araba si sviluppò sotto l'influenza decisiva della tradizione grammaticale greca ed ellenistica. ${ }^{9}$ Nell'opera fondamentale della linguistica araba, Kitâb («Libro»), che Sîbawayhi scrisse già nell'ottavo secolo della nostra era, si trova esposta la concezione della classificazione delle «parti della lingua araba». Fatta eccezione - entro certi limiti - per la relativa terminologia, fino a tutt'oggi questa concezione non ha subito mutamenti. Sîbawayhi descrive tre categorie di parole: nome (ar. «ism»), verbo (ar. «fi ' l») e particella (ar. «harf»), citando esempi tipici per ogni categoria. I nomi che esprimono un'azione egli li mette in una classe speciale, destinguendoli dalle forme verbali finite, ma anche dai nomi. ${ }^{10}$ L'esigenza di separare, almeno for-

8 v. Iu. S. Maslov, op. cit., 202.

9 v. J. B. Fischer, The Origin of Tripartite Dinision of Speech in Semitic Trammar, Jewish Quarterly Review, 53, 1962/63, 1-21; 54, 1963/64, 132-160; I. Lichtenstädter, Encyclopédie de l'Islam, s $\nabla$. «nahw». La teoria dell'origine greca della grammatica araba oggi non è più considerata ineccepibile come un tempo, จ. M. G. Carter, Les origines de la grammaire arabe, Revue des Etudes Islamiques, XL, 1, 1972, 69-97.

10 v. Sîbwayhi, Kitâb ..., Cairo, 1898, 2. I grammatici posteriori non hanno apportato alcuna modifica sostanziale a questa impostazione fondamentale, $v$. ad. es. Al-Zağğâgî, Précis se grammaire arabe, pub. par M. Ben Cheneb, Paris, 1957, 


\section{Darko Tanaskooić}

malmente, il mașdar dai nomi (in base alla distribuzione delle vocali) si può osservare nella maggior parte dei grammatici arabi, il che ha un'importanza determinante per comprendere la concezione che essi hanno di questa categoria e della sua natura. ${ }^{11}$ A questo punto bisogna precisare che mașdar, dal punto di vista lessicale significa «origine», «fonte», esso è pertanto paragonabile ad una sorgente dalla quale scaturiscono diverse manifestazioni concrete dell'azione verbale. Questa interpretazione del mașdar ha ricevuto un'ampia elaborazione nel corso dello sviluppo della grammatica araba, ma esistono anche posizioni diverse.

Possiamo affermare - senza pretese di assolutizzazione - che l'arabistica europea più antica si è basata in gran parte sui punti di vista dei filologi arabi medievali, soprattutto quando viene affrontata la divisione delle parole in categorie, ambito nel quale si nota una forte influenza di concezioni formulate in base alla conoscenza delle lingue indoeuropee. La maggior parte degli arabisti interpreta il mașdar come «l'infinito arabo», il che è ben comprensibile, perché fra il mașdar e l'infinito delle lingue europee esistono molti punti di contatto. ${ }^{12}$ L'arabista russo L. N. Mel'iancev, nel suo interessante articolo intitolato Il mașdar arabo e le questioni della teoria delle parti del discorso, osserva che lo studio del mașdar e dei fenomeni corrispondenti riscontrabili nelle altre lingue, nell'arabistica europea, non poggia su basi scientifiche, perché «tutti i tentativi di riferimento all'analogia funzionale tra il mașdar e l'infinito si riducono al trasferimento da una tradizione linguistica all'altra delle concezoni di categoria». ${ }^{13} \mathrm{Ci}$ sembra che questo giudizio critico non sia

1957,2 , dove si dice che il mașdar è «il nome del verbo» e che «il verbo è derivato da esso», il che dimostra che i grammatici arabi non separano affatto il mașdar dalla più ampia categoria verbale di parole. Il problema del trattamento del mașdar è stato uno dei punti sui quali si scontrarono le posizioni di due scuole classiche della grammatica araba, quella di Kûfa e quella di Başra (v. H. Fleisch, Traité de philologie arabe I, Beyrouth, 1961, 267). I rappresentanti della scuola di Kûfa ritenevano che il masdar derivasse dal verbo, mentre a Baṣra sostenevano che esso fosse l'origine di tutte le forme verbali (su questi temi, come pure sulle altre questioni controverse v. Ibn al- Anbârî, Kitâb al-Insâff ..., ed. G. Weil, Leiden, 1913).

11 v. Il libro dei verbi di Abû Bakr Muhammad B. 'Umar B. 'Abd al-'Azîz Ibn al-Qûtiyya, pub. da I. Guidi, Leida, 1894, 13-14.

12 v. ad. es. W. Wright, $A$ Grammar of the Arabic Language I, Cambridge, 1971, 109-110, «nomina verbi» or «nomina actionis» (infinitives); G. Brockelmann, Arabische Grammatik, Leipzig, 1965, 64; R. Blachère et M. GaudefroyDemombynes, Grammaire de l'arabe classique, Paris, 1952, 77-84; L. Veccia Vaglieri, Grammatica teorico-pratica della lingua araba I, Roma, 1938, 136, «L'infinito arabo è in realtà un sostantivo: sostantivo verbale».

13 v. A. N. M., Arabskij masdar $i$ boprosy teorii častej reči, Vestnik Moskovskogo Universiteta, Vostokovedenie, 1, 1975, 85-91; l'autore dà esaurienti informazioni sui contributi dei linguisti ed arabisti sovietici e russi sul problema della classificazione delle unità linguistice e, particolarmente, sullo studio dell'infinito e del maşdar. Lo scopo che questo scritto si prefigge è quello di «analizzare a fondo i possibili accostamenti teorici allo studio delle parole nominali che esprimono un'azione». 


\section{Linguistica $X V$}

del tutto giustificato, perché gli arabisti europei, per lo più, hanno confrontato l'infinito e il mașdar per motivi «pedagogici», allo scopo, cioè, di avvicinare la forma araba ai fruitori europei dei loro libri, ma senza intenzioni teoriche più profonde. Anzi, alcuni di loro hanno esplicitamente ammesso l'insufficienza presente nella formulazione teorica delle soluzioni che, in mancanza di altre migliori e più adeguate, sono stati costretti ad accettare. ${ }^{14}$ Del resto, a tutt'oggi, non sono stati trovati metodi di spiegazione più felici. Considerate nel loro insieme le opere di grammatica araba sorte fuori dell'area araba, si può concludere che in esse il mașdar, per lo più, viene considerato come un nome verbale, in quanto si dà la precedenza al criterio formale di classificazione su quello semantico, mentre l'aspetto funzionale è tuttora insufficientemente elaborato, sebbene offra possibilità molto interessanti (soprattutto se considerato alla luce della posizione del distribuzionalismo).

La grammatica comparata delle lingue semitiche non ci può essere di grande aiuto, perché questa disciplina si muove sui sentieri noti e battuti della linguistica comparativa classica, il che è, entro certi limiti, comprensibile perché questo ramo della comparativistica si è formato relativamente tardi. ${ }^{15}$ Negli studi più noti dedicati alla grammatica comparata delle lingue semitiche, il mașdar viene considerato come infinito e come nome verbale e come tale viene messo in un'unica categoria insieme con altre forme di lingue semitiche dalle quali si distingue per molte caratteristiche essenziali senza che venga affrontata un'analisi più profonda delle somiglianze e delle differenze. ${ }^{16}$ Un patricolare interesse l'ha suscitato l'infinito ebraico nel quale le caratteristiche verbali e nominali sono in certa misura divise formalmente in quanto esistono due forme d'infinito, una delle quali (infinitivus absolutus) presenta più accentuate le caratteristiche verbali, mentre l'altro (infinitivus constructus) quelle nominali. ${ }^{17}$ Un progresso significativo è stato quello portato dal libro del noto semitista russo B. M. Grande Introduzione allo studio comparato delle lingue semitiche, nel quale l'autore considera particolarmente quelle categorie di parole delle lingue semitiche che non si possono includere

14 Così, ad esempio, R. Blachère e M. Gaudefroy-Demombỳnes (op. cit., 77), affrontando la trattazione delle parole nominali della lingua araba annunciano: «Pertanto, qui ci accontenteremo di comporre un elenco che non apparirà sempre poggiato su basi logiche». Abbiamo scelto il «riconoscimento» citato, perché fra le parole nominali, al primo posto della lista stessa, troviamo proprio i masdar. 234.

15 v. C. Tagliavini, Panorama di storia della linguistica, Bologna, 1970, 233-

${ }^{16}$ v. C. Brockelmann, Grundriss der pergleichenden Grammatik der semitischen Sprachen I, Berlin, 1908, 578; Idem, Kurzgefasste vergleichende Grammatik der semitischen Sprachen, Berlin, 1908, 273-276; L. H. Gray, Introduction to Semitic Comparatioe Linguistics, New York, 1934, 100-102; An Introduction to the Comparative Grammar of the Semitic Languages, by S. Moscati, A. Spitaler, E. Ullendorf, W. van Soden, Wiesbaden, 1964, 146-147.

${ }^{17}$ Esaurienti dati bibliografici su queste opere, per lo più superate, in L. H. Gray, op. cit. 


\section{Darko Tanaskooić}

nelle due classi fondamentali, quella del nome e quella del verbo, ma si trovano a metà fra queste due come tipi di «transizione» (russo: »promežutočnye»). L'autore esamina poi dettagliatamente tali categorie di parole, fra di esse anche il mașdar arabo, del quale dice che "per significato è vicino all'infinito, mentre per forma ed uso è un nome». ${ }^{18}$ Grande, insistendo sulle due categorie fondamentali di parole, assume una posizione di principio che si pone in antitesi nei confronti della tendenza formalistica verso la moltiplicazione del numero delle classi delle unità linguistiche che permette una soluzione soltanto apparente delle difficoltà tassonomiche. Tempo fa, molto prima di Grande, manifestò un'idea analoga anche il grande studioso tedesco Jacob Grimm ${ }^{19}$ e su questa linea si trovano anche tutte le altre richieste che nella suddivisione delle parole in categorie oltre al criteri strettamente formali vengano costantemente impiegati anche quelli funzionali e soprattutto quelli semantici, giacché, come ha scritto Frank Palmer, «come linguisti noi dobbiamo compilare le nostre grammatiche in modo che esse siano collegate con la semantica tanto quanto con la fonetica: la nostra grammatica deve essere sensibile alla semantica». ${ }^{20}$

Interessante è il materiale che offre l'analisi del trattamento dell'infinitivo e delle forme a questa simili che si trova nella sistematizzazione grammaticale data di altre lingue, perché l'infinito pone il classificatore davanti a problemi analoghi a quelli suscitati dal mașdar. Le soluzioni adottate nelle singole suddivisioni dipendono direttamente dalle caratteristiche che presenta l'infinito nella struttura della lingua che si analizza. Se prevalgono quelle formali proprie dei nomi i linguisti tendono a classificare l'infinito fra i nomi, ma, in modo caratteristico, spiegano questa

18 B. M. Grande Voedenie o sraonitel'noe izučenie semitskih jazykoo, Moskva, 1972, 186-190. Analogamente a Grande, anche l'eminente arabista A. A. Kovalev ritiene che «il masdar arabo rappresenti una categoria intermedia (sincretica), che riunisce in se elementi del nome e del verbo», v. Vyraženie kategorii oremeni o sooremennom arabskom jazyke, Moskva, 1950, 221 (secondo A. N. Mel'ancev, op. cit. 88). Nella linguistica, del resto, si è già affermato il termine «parti miste del discorso» (ingl. «mixed parts of speech»), ma la soluzione di Grande, a nostro avviso, è migliore, perché non è statica, ma esprime la dinamica degli spostamenti che si verificano all'interno di una lingua.

19 v. J. G., Deutsche Grammatik IV, Gütersloh 1898, 1, «in realtà esistono soltanto due categorie di parole (zwei Wortarten), nomina e verba»; sul problema delle categorie delle parole nella lingua tedesca v. J. Djukanović, Vrste reči $u$ nemačkom jeziku $u$ soetlosti sapremene lingoistike, Anali Filološkog fakulteta, $\mathrm{X}$, Beograd, 1970, 355-370. Alla fondamentale dicotomia nome-verbo fa riferimento anche A. Meillet, v. Linguistique historique et linguistique générale, Paris, $1921,175-176$, e perfino E. Sapir, che è estremamente scettico nei confronti di ogni tentativo di formulazione di una divisione universale delle parole in categorie, conclude che non c'è lingua che non conosca due classi fondamentali: il nome e il verbo, v. Le langage, Paris, $1970,116$.

20 v. F. P., Grammar, Harmondsworth, 1971, 40. Anche D. Skiljan, nell'articolo, per molti aspetti interessante, Lingoistika $i$ marksizam, Dometi, 8, 8, 1975, 5-11, sostiene la necessità di riconoscere una maggiore importanza al «piano del contenuto linguistico»e al «campo semantico». 
inclusione con il processo definito di sostantivazione dell'infinito, il che dimostra che, anche in questi casi, l'infinito è pur sempre considerato una categoria verbale di parole. Nella lingua russa, ad esempio, nella quale l'infinito non possiede un numero rilevante di proprietà formali del nome, ma presenta un comportamento nominale soltanto sul piano sintattico, ha prevalso la classificazione dell'infinito come forma verbale, sistemazione alla quale contrubuisce anche l'esistenza in questta lingua di nomi verbali chiaramente definiti. ${ }^{21}$ Anche la grammatica italiana classifica l'infinito fra le forme verbali, insieme con il gerundio e il participio, sebbene la sostantivazione dell'infinito in italiano sia un fenomeno molto ampio..$^{22}$ La lingua turca contemporanea possiede alcune forme che esprimono astrattamente il significato dell'azione verbale e che per lo più si spiegano come nomi verbali. Una distinzione essenziale fra di esse viene compiuta in base al grado di nominalizzazione, vale a dire in base all'utilizzazione di un criterio semantico che è naturalmente seguito dalla registrazione delle corrispondenti proprietà morfologiche e sintattiche. ${ }^{23}$ Si potrebbe citare ancora tutta una serie di esempi tratti da tradizioni grammaticali nazionali diverse, ma già da quelli ricordati emerge un dato fondamentale, le complesse difficoltà davanti alle quali le parole di tipo «transizionale» (usiamo il termine di Grande) pongono i linguisti classificatori.

Da quanto sopra esposto deriva che al mașdar (come del resto ad ogni altro fenomeno linguistico), quando se ne tenti la classificazione, bisogna accostarsi con molta prudenza e considerando la lingua nel suo complesso. Questo procedimento è necessario soprattutto nel caso dell'arabo, il cui carattere flessivo fortemente accentuato "costituisce una materia molto difficile per la sistemazione delle unità linguistiche».24 Per dividere le parole categorie è necessario fissare una gerarchia di criteri classificatori. Una tale gerarchia esiste implicitamente all'interno di ogni sistema completo di divisione delle unità linguistiche, ma è necessario esprimerla esplicitamente ed applicarla nel modo più coerente possibile. È chiaro che la gerarchia metodologica dei criteri non sottintende in nessun

21 v. A. N., Mel'iancev, op. cit. 87; Grammatika sooremennogo russkogo literaturnogo jazyka, Moskva, 1970, 417-418.

${ }_{22}^{2}$ v. M. Regule e J. Jernej, Grammatica italiana descrittioa su basi storiche e psicologiche, Bern, 1965, 225, «forma nominale del'verbo, l'infinito esprime il senso verbale nel modo più chiaro assumendo però spesso non solo le funzioni, ma anche il valore di un sostantivo»; L. Satta, La prima scienza, Messina-Firenze, 1975, 324-325.

${ }^{23}$ v. G. L. Lewis, Turkish Grammar, Oxford, 1969, 167-173; E. Rossi, Manuale di lingua turca I, Roma, 1963, 109-113.

${ }^{24}$ v. A. Amirova, B. A. Ol'hovnikov, Iu. V. Roždestvenski, Očerki po istorii lingoistiki, Moskva, 1975, 150; opinione analoga esprimono anche R. Blachère e M. Gaudefroy-Demombynes, op. cit. 77 . Nessuna meraviglia che nel caso delle lingue flessive $i$ linguisti si accontentino spesso di una divisione tripartita delle parti del discorso. 


\section{Darko Tanasković}

modo una valutazione gerarchica delle classi delle unità linguistiche. La divisione fondamentale e naturale delle caratteristiche linguistiche rilevanti dovrebbe considerare le parole sotto due angoli visivi, quello formale e quello contenutistico, ${ }^{25}$ pur conservando la precedenza al criterio del contenuto categoriale, naturalmente soltanto dal punto di vista metodologico. Se ora ritorniamo alla suddivisione data da Aristotele e alla «macro-divisione» delle parole in categorie che troviamo nella grammatica araba classica, dovremmo annoverare il masdar fra le forme verbali, perché esso presenta una semantica dell'azione chiaramente espressa. Nei limiti di una primaria divisione generale è necessario, poi, sviluppare un'analisi completa del mașdar dal punto di vista formale e funzionale, tenendo esclusivamente presenti le funzioni del mașdar all'interno della struttura linguistica. Nel corso di questa analisi secondaria bisogna descrivera dettagliatamente tutti i tipi possibili delle funzioni del mașdar e pravalentemente quelle che, dal punto di vista formale, definiscono il mașdar come categoria nominale di parole e che, nella determinazione semantica fondamentale possono far sì che, con l'evolversi della lingua, il mașdar si avvicini alla classe generale delle parolé nominali, fino ad inserirsi in essa, perdendo la capacità d'interpretazione della dinamica dell'azione verbale. In questo modo si può gettare un ponte sopra l'abisso artificialmente creato fra il piano del contenuto e quello della forma nella sistematizzazione delle unità linguistiche. Questo processo di «nominalizzazione» lo possiamo oservare bene nel caso del mașdar, anzi esso è stato da tempo indicato come la sua caratteristica essenziale (come nel caso dell'infinito nella maggior parte delle altre lingue). ${ }^{26}$ Una tendenza più marcata alla sostantivazione si può notare quando ad una sola radice verbale sono legate più forme del mașdar. Di norma un mașdar conserva caratteristiche verbali, mentre l'altro (per lo più si tratta di due) si sposta verso quelle del nome.

Valide conoscenze di ordine pratico e teorico si possono acquisire dallo studio del mașdar nel ruolo di oggetto o di complemento, cioè di completamento dell'azione di un altro verbo, funzione nella quale esso si manifesta come l'equivalente delle proposizioni dichiarativa e finale che possono essere formulate anche analiticamente, vale a dire per mezzo della congiunzione e della forma verbale personale del modo verbale adeguato, per lo più del congiuntivo. In questa sua funzione il mașdar è simile all'infinito del maggior numero di lingue di famiglie linguistiche diverse. Così, ad esempio, la frase «voglio andare» si può esprimere in due modi: usando il mașdar - urîdu ad-dihâba (ad-dihaba $=$ articolo determinativo + mașdar + accusativo), oppure con la forma personale del verbo finito - urîdu'an adhaba (an a $\underline{d h a b a}=$ congiunzio

25 v. A. N. Mel'iancer, op. cit. 89.

26 v. particolarmente B. M. Grande, Kurs arabskoj grammatiki o sraonitel'noistoričeskom osveščenii, Moskva, 1963, 189-192, 253-254. 
ne + verbo al congiuntivo). ${ }^{27}$ Sebbene il mașdar in questi casi abbia tutte le caratteristiche del nome (articolo determinativo, la declinazione per casi, la funzione di oggetto dell'azione verbale) è assai evidente che esso svolge la funzione di esprimere il significato verbale. Nel momento in cui il massdar, come equivalente di una proposizione oggettiva, non può più soddisfare, ovvero quando il suo impiego come oggetto o come complemento comincia a disturbare una sensibilità linguistica corretta, si può parlare dello spostamento del mașdar verso la classe nominale delle parole. La natura proteica del mașdar si può individuare soltanto attraverso una descrizione ed un'analisi esauriente della sua posizione funzionale all'interno della struttura linguistica. Allo stesso tempo non si può perdere di vista la sua fondamentale classificazione come categoria verbale di parole. Ogni rigida divisione formale deforma l'immagine della realtà linguistica e non può in alcun modo misurarsi efficacemente con problemi quali sono quelli suscitati dalla classificazione del mașdar arabo. Un accostamento corretto e completo al mașdar, come pure alle altre catagorie di parole, oltre a quella teorica, ha certamente un'importanza pratica nell'ambito della linguistica applicata, soprattutto al fine di perfezionare la metodologia dell'insegnamento della lingua araba, perché molte delle soluzioni alle quali si ricorreva in questo campo (e alle quali si ricorre tutt'ora) non solo non facilitano la comprensione delle caratteristiche essenziali della struttura linguistica, ma, all'incontrario, deformano in misura considerevole l'immagine reale, costringendo la lingua araba in schemi estranei alla sua sostanza e alla sua natura.

\section{Rezime}

\section{ARAPSKI MASSDAR U SVETLOSTI TEORIJE O VRSTAMA RECI}

Podela jezičkih jedinica na vrste ili klase stara je koliko i sama lingvistika. $\mathrm{Na}$ njenom usavršavanju predano $i$ uporno je rađeno tokom čitave istorije nauke o jeziku, a kroz taj rad došlo se do čitavog niza značajnih opštelingvističkih saznanja. Tradicionalna podela reči na vrste potiče još od antičkih filosofá, prvenstveno Aristotela, a izgrađivana je uglavnom u funkciji sistematizovanja građe koju so pružali indoevropski jezici. lako su do danas stvoreni različiti tipovi klasifikacije, od logičkih do krajnje formalističkih, može se reći da potpuno zadovoljavajuća rešenja u ovoj složenoj oblasti nisu mogla biti pronađena, jer je po pravilu prednost kod svake pojedine klasifikacije davana jednoj određenoj grupi kriterijuma, morfološkim, sintaksičkim ili semantičkim, a ređ̃e i funkcionalnim. U celini se može zapaziti dominacija formalnih kriterijuma.

${ }^{27} \mathrm{La}$ situazione descritta è vicina all'uso alternativo delle construzioni analitiche e sintetiche, cioè delle forme finite del verbo e dell'infinito, verificabile nel serbocroato, ad. es. „Želim da idem = Želim ići (v. M. Stevanović, Saoremeni srpskohrnatski jezik, II, Beograd, 1974, 765). Nell'arabo l'uso dell'infinito oggettivo è notevolmente più ampio che nella maggior parte delle lingue nelle quali compare come modo possibile di completamento dell'azione espressa dal verbo. 


\section{Darko Tanaskooić}

$\mathrm{Na}$ primeru »maşara«, oblika iz arapskog jezika, koji nosi i glagolske i imenske osobine i obeležja, moguce je sagledati neke od problema podele reči na vrste, kao i nemoć izvesnih tradicionalnih klasifikacionih sistema. »Masdar" je blizak infinitivu i glagolskoj imenici indoevropskih jezika, pa je u svetlu te analogije uglavnom i razmatran, kako u arabistici, tako $i$ šire, u semistici, bez potrebne analize njegove stvarne prirode. Klasična arapska gramatička nauka posmatra »maşdar kao glagolsku reč u okviru svoje tripartitne podele reči na vrste (ime - glagol - čestica), ali ga donekle $\mathrm{i}$ izdvaja iz razloga formalne i semantičke specifičnosti ovog oblika. »Maşdar« i druge slične jezičke jedinice sinkretičkog karaktera upućuju na potrebu uspostavljanja izvesne hijerarhije klasifikacionih kriterijuma, tačnije, dva nivoa posmatranja, pri čemu bi prednost trebalo dati kategorijalnom sadržaju pojedine reči, tj. njenoj glagolskoj ili imenskoj semantici, a zatim bi u granicama te primarne podele trebalo izvršiti što iscrpniju i svestraniju formalnu i funkcionalnu analizu. Rezultati te, u metodološkom smislu sekundarne operacije, mogu svojom snagom delovati i na odnose na stepenu primarne podele, kao odraz stalnih unutarjezičkih pomeranja i dijalektičke veze između plana sadržaja i plana oblika u jeziku. 